\title{
AN RF CAVITY FOR THE NLC DAMPING RINGS*
}

\author{
R.A. Rimmer ${ }^{\dagger}$, D. Atkinson, J.N. Corlett, G. Koehler, D. Li, N. Hartman, \\ J. Rasson, T. Saleh, R. Weidenbach, LBNL, Berkeley, CA 94720, USA
}

\begin{abstract}
We report on the design and development of a strongly HOM damped copper RF cavity for the NLC damping rings. The cavity is based on the successful PEP-II RF cavity but incorporates many simplifications and improvements. The cavity is designed for a frequency of $714 \mathrm{MHz}$, gap voltage of $500 \mathrm{kV}$ and beam current of 800 $\mathrm{mA}$. We present the RF design and HOM impedance calculations done in MAFIA, the RF, thermal and stress analyses performed in ANSYS and the simplified mechanical design and assembly process. Designs for the RF window, HOM loads and tuners are described. Options for increasing the stored energy or further lowering the HOM impedance are discussed. This design could easily be scaled up or down in frequency and could be useful for other projects such as new light sources.
\end{abstract}

\section{INTRODUCTION}

We have developed a design for the damping ring RF cavities, figure 1, based on the successful PEP-II design. In order to provide the required voltage and stable beam at high currents the cavities must have strong HOM damping, a high gradient and heavy beam loading. Table 1 shows some parameters of the damping ring and predamping ring (PDR) RF systems. The total voltage and beam power can be delivered by three copper cavities in each of the damping rings but seven cavities are needed in the positron pre-damping ring. The maximum bunch frequency is now the same as the RF frequency, at 714 $\mathrm{MHz}$ (previously only every other bucket was filled). The power density and stresses in the NLC cavity remain comparable to the current PEP-II operating conditions (taking the scaled PEP-II design as the baseline). Means of reducing the stress without sacrificing the good HOM damping properties and accelerating mode efficiency have been investigated. Simplifications of the mechanical design and fabrication have also been developed. [1,2] The availability of better analysis tools also now allows more detailed study of some aspects of the design.

\section{RF DESIGN}

The basic cavity design is a conventional reentrant shape except for the addition of the HOM damping ports and making the center section spherical rather than torroidal. The spherical mid section has little effect on the shunt impedance but simplifies the port machining and reduces the cost. A torroidal body or even a plain cylinder necessitates machining complex curves for the blend or intersection of the ports, requiring multi-axis machining. The profile change redistributes the HOM fields slightly so the optimum HOM port location was re-checked.

\footnotetext{
*This work was supported by the US. Department of Energy under contracts DE-AC03-76SF00098. † RARimmer@lbl.gov
}

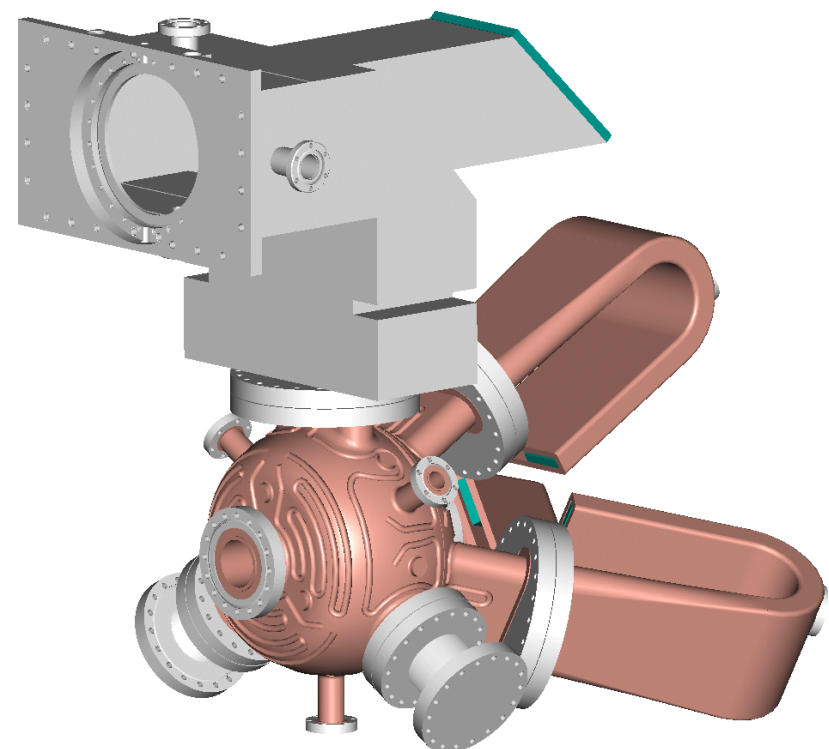

Figure 1. CAD model of full cavity assembly.

Table 1. Damping ring RF parameters.

\begin{tabular}{|l|c|c|}
\hline & PDR & Damping ring \\
\hline Beam Current & $0.75 \mathrm{~A}$ & $0.80 \mathrm{~A}$ \\
\hline $\mathrm{V}_{\mathrm{RF}}$ & $3.4 \mathrm{MV}$ & $1.07 \mathrm{MV}$ \\
\hline Number of Cavities & 7 & 3 \\
\hline Shunt Impedance* & $3.0 \mathrm{M} \Omega$ & $3.0 \mathrm{M} \Omega$ \\
\hline Cavity Wall loss & $39.3 \mathrm{~kW}$ & $22.4 \mathrm{~kW}$ \\
\hline Beam Power & $478 \mathrm{~kW}$ & $602 \mathrm{~kW}$ \\
\hline Klystron Power & $1 \mathrm{MW}$ & $1 \mathrm{MW}$ \\
\hline Coupling Factor & 2.7 & 10 \\
\hline \multicolumn{2}{|c|}{$* \mathrm{~V}^{2} / 2 \mathrm{P}$, assuming $70 \%$ of calculated $\mathrm{Q}_{0}$}
\end{tabular}

The HOM port profile was changed from the rectangular slot used for PEP-II to a narrower, slightly ridged, and rounded "dumbbell" profile, figure 2. This HOM port shape reduces the local heating where it joins the cavity and eliminates the need for an iris at that location. It also leads to mechanical simplifications in the assembly.

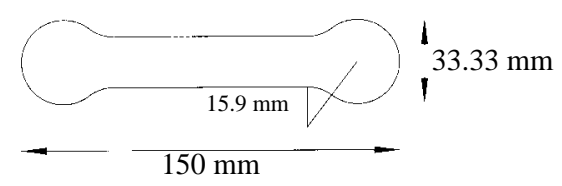

Figure 2. Rounded "dumbbell" HOM-port cross section.

Careful analysis of the HOM damping properties using the latest time domain methods in MAFIA [3], showed that the optimum port location was at an angle of $30^{\circ}$ to the cavity axis (the same as the original PEP-II design). Figure 3 shows the calculated longitudinal impedance spectrum for this cavity. Note that the impedance is well below the expected thresholds for coupled bunch 
instabilities in the damping rings, but close to threshold for the PDR. Further study will indicate whether bunchby-bunch feedback or further HOM loading will be necessary in this case. Figure 4 shows the transverse impedance spectrum. There are a few modes above threshold in all cases, however the growth rates are expected to be lower than or comparable to those driven by the resistive wall impedance and a transverse bunch by bunch feedback system can be used to stabilize them.

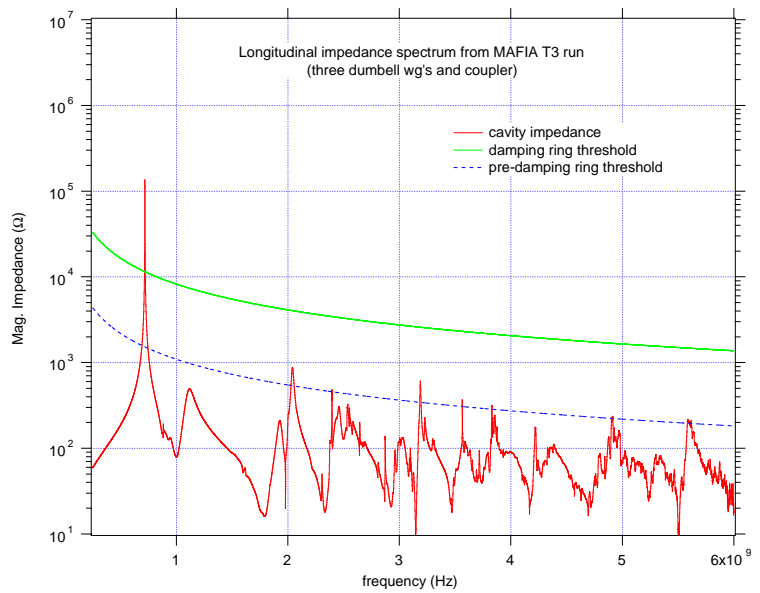

Figure 3. Longitudinal impedance spectrum with approximate thresholds ( 3 cav. DR, 7 cav. PDR).

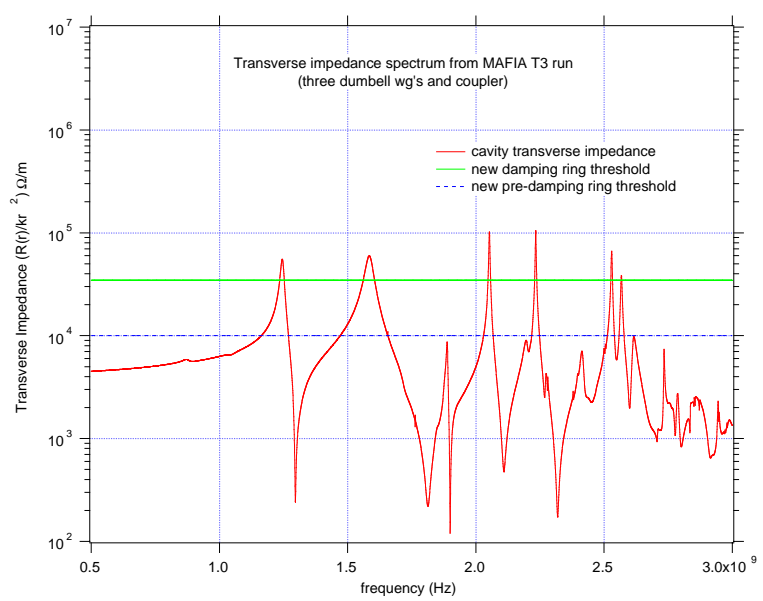

Figure 4. Transverse impedance spectrum with approximate thresholds ( 3 cav. DR, 7 cav. PDR).

\section{MECHANICAL DESIGN}

The mechanical design and fabrication follow the methods used for PEP-II but with some significant simplifications. The surface power density is comparable to PEP-II so a similar cooling channel density is used. The changes to the HOM port shape reduce the local stresses and simplify fabrication. Figure 5 shows the wall power density calculated by ANSYS for one sixth of the geometry, including one HOM port and the coupler. Figure 6 shows the Von Mises stress in the body. The maximum is about $35 \mathrm{MPa}(5000 \mathrm{Psi})$ at $42 \mathrm{~kW}$, safely less than the endurance limit at 10,000 cycles of $\sim 124$ MPa (18,000 Psi) and less than the estimated PEP-II operating stress of $\sim 50 \mathrm{MPa}$ (7300 Psi), at $100 \mathrm{~kW}$.

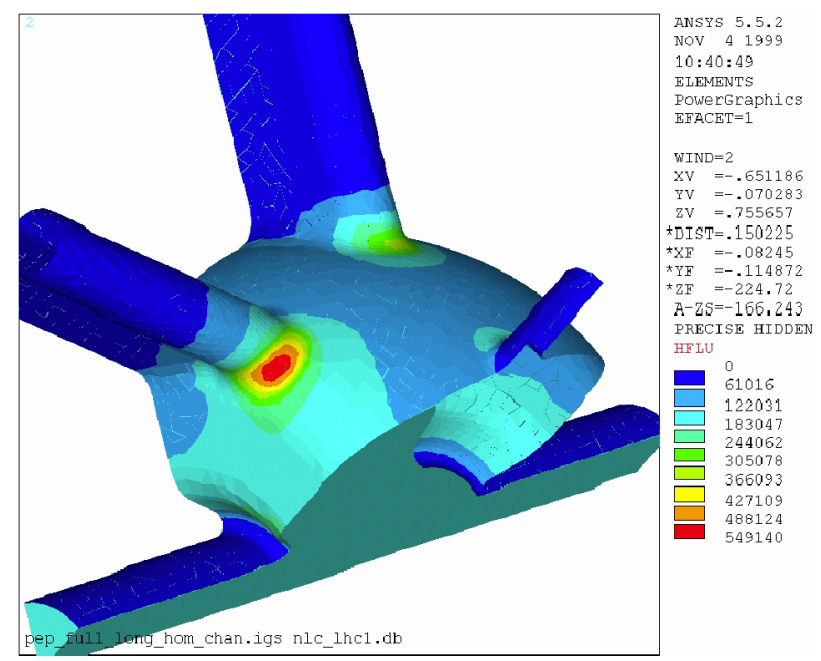

Figure 5. Surface power loss on proposed NLC cavity.

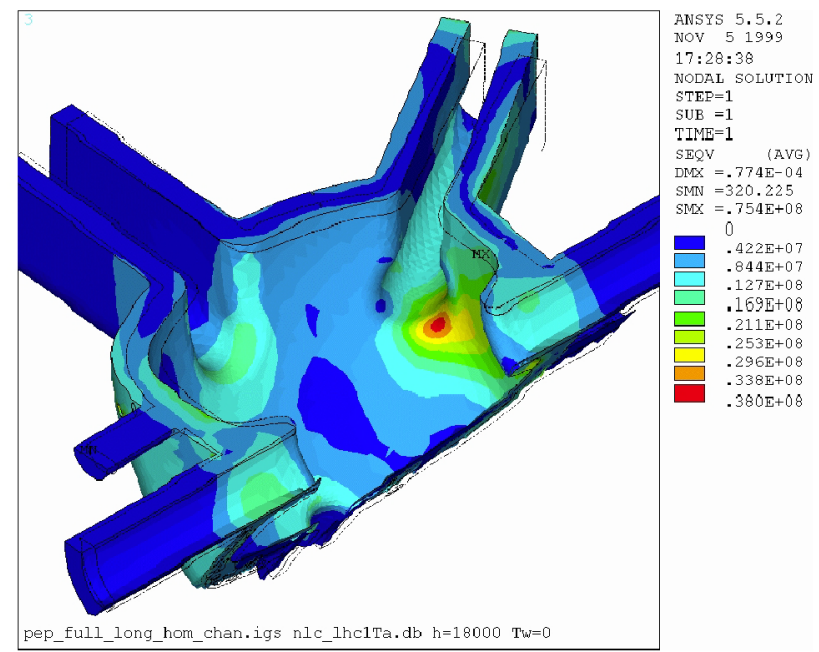

Figure 6 . Von Mises stress in the cavity body.

The cavity will be made from two main forgings, figures 7 and 8, to minimize the number of joining operations. The ports will be machined directly into the forgings and the smooth blend will be finished by the plunge EDM method, figure 9, which is cheaper than multi-axis machining and leaves a good surface finish. After the body is welded all water passages will be covered in one operation by conventional copper plating.

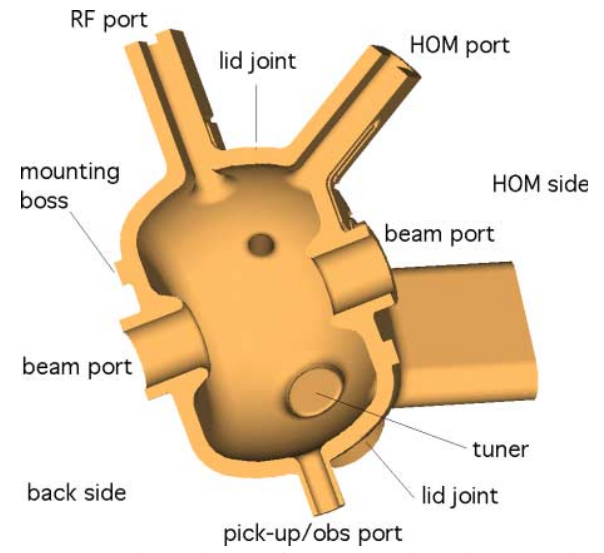

Figure 7. Section through two main pieces of cavity. 

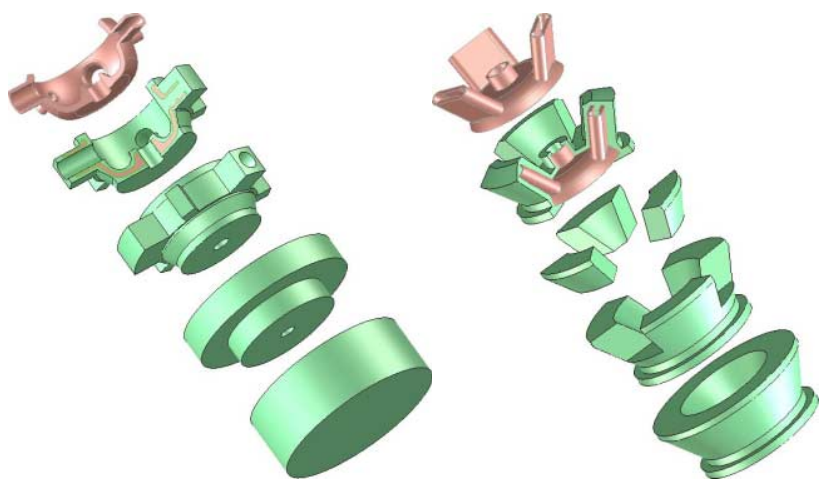

Figure 8 . Development of body and lid from forgings.

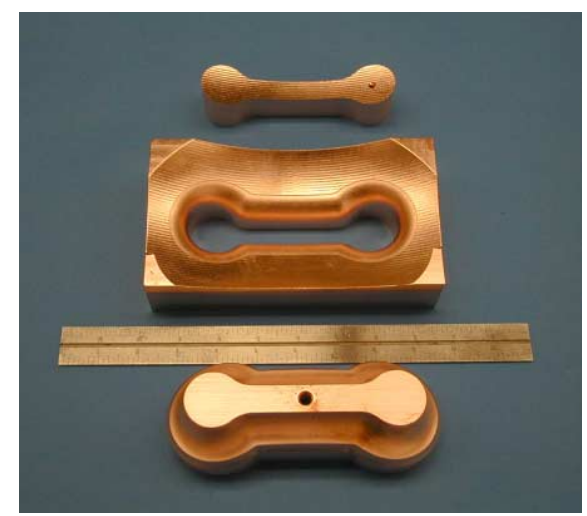

Figure 9. Plunge EDM sample with tellurium copper electrode (bottom) and wire burnout (top).

\section{RF WINDOW, HOM LOADS \& TUNERS}

A high power window, figure 10, has been developed at LBNL for this application and for LEDA, and has been tested to over $800 \mathrm{~kW} \mathrm{CW}$ at LANL [4,5]. The high coupling factor (up to 10), can be achieved by using the same cross section for the coupling iris as for the HOM port.

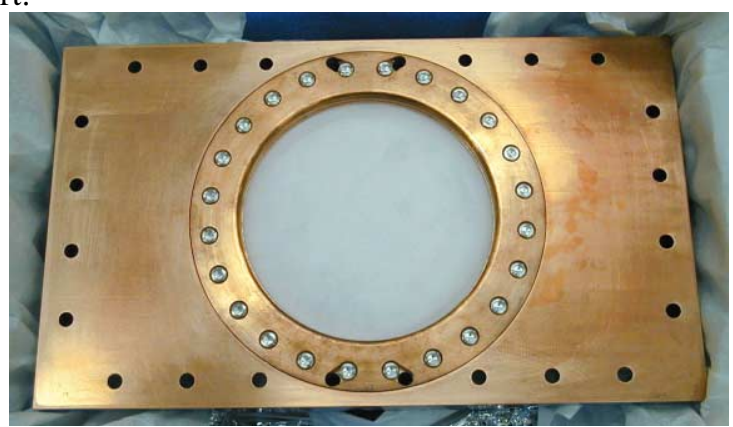

Figure 10. Finished window in waveguide flange.

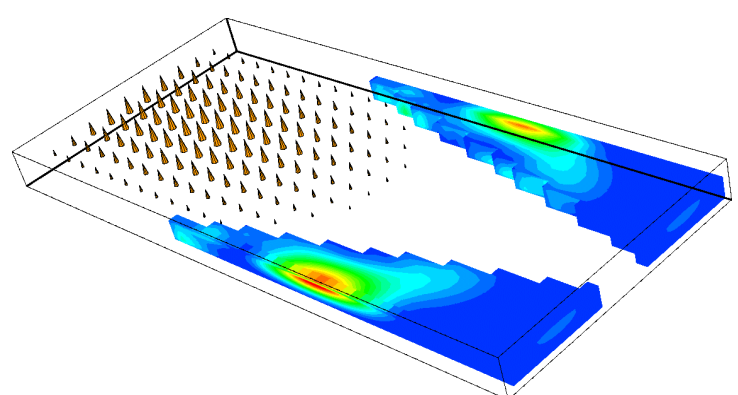

Figure 11. HOM load MAFIA simulation at $1 \mathrm{GHz}$.
The HOM loads are scaled from the PEP-II type and have a calculated VSWR of less than 2:1 from $1 \mathrm{GHz}$ to over $5 \mathrm{GHz}$. Figure 11 shows the distribution of electric field and dielectric losses in the load at $1 \mathrm{GHz}$.

A plunger type tuner with internal cooling will be used, see figure 12. Spring finger contacts may be used at the bottom of the tuner port to protect the vacuum bellows if needed. A second fixed tuner will be used to make final frequency adjustments after welding.

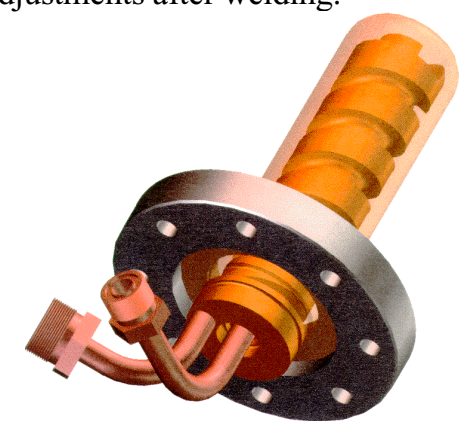

Figure 12. Plunger type tuner with spiral cooling channel.

\section{LOW HOM AND LOW R/Q OPTIONS}

Further HOM damping can be achieved by modifying the existing waveguides or adding additional ones. Schemes with four or six waveguides have been simulated and show good results. It may be desirable to increase the stored energy to reduce the beam induced transients in the rings. This can be achieved by lowering the $\mathrm{R} / \mathrm{Q}$, at the cost of increased RF power, or raising the effective $\mathrm{Q}$ by using an external energy storage cavity. Simulations and cold test models show the most gain from the latter.

\section{CONCLUSIONS}

We have developed a cavity for the damping rings based upon the successful PEP-II design but with better HOM damping, lower stresses and much simpler fabrication. We have a proven RF window design and HOM load and tuner designs that are closely based on existing technology. Options exist for further lowering the HOM impedance or raising the stored energy if required. This design could easily be scaled down in frequency for use in future light sources or other storage rings.

\section{REFERENCES}

[1] "RF Cavity R\&D at LBNL for the NLC Damping Rings, FY1999", R.A. Rimmer et. al., LBNL-45135, CBP Tech Note 196, LCC-0033, Nov. 1999.

[2] "RF Cavity R\&D at LBNL for the NLC Damping Rings, FY2000/2001", R.A. Rimmer et. al., LBNL47949, CBP Tech Note 231, 2001.

[3] "Beam Impedance Calculation and Analysis of Higher Order Modes (HOMs) in Strongly Damped RF Cavities Using MAFIA in the Time Domain", D. Li et. al., Proc. PAC 2001, Chicago, LBNL-48173.

[4] "A High-Power L-Band RF Window", R.A. Rimmer et. al., Proc. PAC 2001, Chicago, LBNL-47968, LAUR 01-2574.

[5] "700 MHz Window R\&D at LBNL", R. Rimmer et. al., 11/2000, LBNL-47939, LAUR 01-2574. 